\title{
Strings and black holes
}

\section{Thibault Damour}

Institut des Hautes Etudes Scientifiques, 91440 Bures-sur-Yvette, France

damour@ihes.fr

Received 15 November 1999, accepted dd.mm.yyyy by ue

Abstract. I review some recent work (done in collaboration with G. Veneziano) which clarifies the existence of a correspondence between self-gravitating fundamental string states and Schwarzschild black holes. The main result is a detailed calculation showing that selfgravity causes a typical string state of mass $M$ to shrink, as the string coupling $g^{2}$ increases, down to a compact string state whose mass, size, entropy and luminosity match (for the critical value $\left.g_{c}^{2} \sim\left(M \sqrt{\alpha^{\prime}}\right)^{-1}\right)$ those of a Schwarzschild black hole. This confirms the idea that the entropy of black holes can be accounted for by counting string states, and suggests that the level spacing of the quantum states of Schwarzschild black holes is exponentially small, and very much blurred by radiative effects.

Keywords: fundamental strings, black holes

PACS: $11.25 .-\mathrm{w}, 04.70 . \mathrm{Dy}$ 


\section{Introduction}

This contribution deals with the "explanation" of the quantum properties of black holes (and, in particular, the statistical meaning of black hole entropy) hopefully provided by string theory. Most of the stringy literature has concentrated (for reasons recalled below) on some special, supersymmetric extreme black holes (BPS black holes). [These black holes carry special (Ramond-Ramond) charges and their microscopic structure seem to be describable in terms of Dirichlet-branes.] By contrast, we consider here the simplest, Schwarzschild black holes (in any space dimension $d$ ). It will be argued that their "microscopic structure" involves only fundamental string states. However, the lack of supersymmetry means that it becomes essential to deal with self-gravity effects.

To start with, let us recall that thirty years ago the study of the spectrum of string theory revealed [1] a huge degeneracy of states growing as an exponential of the mass. A few years later Bekenstein [2] proposed that the entropy of a black hole should be proportional to the area of its horizon in Planck units, and Hawking [3] fixed the constant of proportionality after discovering that black holes do emit thermal radiation at a temperature $T_{\mathrm{Haw}} \sim R_{\mathrm{BH}}^{-1}$.

When string and black hole entropies are compared one immediately notices a striking difference: string entropy is proportional to the first power of mass in any number of spatial dimensions $d$, while black hole entropy is proportional to a $d$-dependent power of the mass, always larger than 1. In formulae:

$$
S_{s} \sim \frac{\alpha^{\prime} M}{\ell_{s}} \sim M / M_{s} \quad, \quad S_{\mathrm{BH}} \sim \frac{\text { Area }}{G_{N}} \sim \frac{R_{\mathrm{BH}}^{d-1}}{G_{N}} \sim \frac{\left(g^{2} M / M_{s}\right)^{\frac{d-1}{d-2}}}{g^{2}},
$$

where, as usual, $\alpha^{\prime}$ is the inverse of the classical string tension, $\ell_{s} \sim \sqrt{\alpha^{\prime} \hbar}$ is the quantum length associated with it $\mathrm{D}, M_{s} \sim \sqrt{\hbar / \alpha^{\prime}}$ is the corresponding string mass scale, $R_{\mathrm{BH}}$ is the Schwarzschild radius associated with $M$ :

$$
R_{\mathrm{BH}} \sim\left(G_{N} M\right)^{1 /(d-2)},
$$

and we have used that, at least at sufficiently small coupling, the Newton constant and $\alpha^{\prime}$ are related via the string coupling by $G_{N} \sim g^{2}\left(\alpha^{\prime}\right)^{(d-1) / 2}$ (more geometrically, $\left.\ell_{P}^{d-1} \sim g^{2} \ell_{s}^{d-1}\right)$.

Given their different mass dependence, it is obvious that, for a given set of the fundamental constants $G_{N}, \alpha^{\prime}, g^{2}, S_{s}>S_{\mathrm{BH}}$ at sufficiently small $M$, while the opposite is true at sufficiently large $M$. Obviously, there has to be a critical value of $M$, $M_{c}$, at which $S_{s}=S_{\mathrm{BH}}$. This observation led Bowick et al. [4] to conjecture that large black holes end up their Hawking-evaporation process when $M=M_{c}$, and then transform into a higher-entropy string state without ever reaching the singular zeromass limit. This reasoning is confirmed [5] by the observation that, in string theory, the fundamental string length $\ell_{s}$ should set a minimal value for the Schwarzschild

\footnotetext{
${ }^{a}$ As we shall discuss, the self-interaction of a string lifts the huge degeneracy of free string states. One then defines the entropy of a narrow band of string states, defined with some energy resolution $M_{s} \lesssim \Delta E \ll M$, as the logarithm of the number of states within the band $\Delta E$.

${ }^{\mathrm{b}}$ Below, we shall use the precise definition $\ell_{s} \equiv \sqrt{2 \alpha^{\prime} \hbar}$, but, in this section, we neglect factors of order unity.
} 
radius of any black hole (and thus a maximal value for its Hawking temperature). It was also noticed [4], [6], [7] that, precisely at $M=M_{c}, R_{\mathrm{BH}}=\ell_{s}$ and the Hawking temperature equals the Hagedorn temperature of string theory. For any $d$, the value of $M_{c}$ is given by:

$$
M_{c} \sim M_{s} g^{-2} .
$$

Susskind and collaborators [6], [8] went a step further and proposed that the spectrum of black holes and the spectrum of single string states be "identical", in the sense that there be a one to one correspondence between (uncharged) fundamental string states and (uncharged) black hole states. Such a "correspondence principle" has been generalized by Horowitz and Polchinski [9] to a wide range of charged black hole states (in any dimension). Instead of keeping fixed the fundamental constants and letting $M$ evolve by evaporation, as considered above, one can (equivalently) describe the physics of this conjectured correspondence by following a narrow band of states, on both sides of and through, the string $\rightleftharpoons$ black hole transition, by keeping fixed the entropy $S=S_{s}=S_{\mathrm{BH}}$, while adiabatically ${ }^{2}$ varying the string coupling $g$, i.e. the ratio between $\ell_{P}$ and $\ell_{s}$. The correspondence principle then means that if one increases $g$ each (quantum) string state should turn into a (quantum) black hole state at sufficiently strong coupling, while, conversely, if $g$ is decreased, each black hole state should "decollapse" and transform into a string state at sufficiently weak coupling. For all the reasons mentioned above, it is very natural to expect that, when starting from a black hole state, the critical value of $g$ at which a black hole should turn into a string is given, in clear relation to (3), by

$$
g_{c}^{2} M \sim M_{s}
$$

and is related to the common value of string and black-hole entropy via

$$
g_{c}^{2} \sim \frac{1}{S_{\mathrm{BH}}}=\frac{1}{S_{s}} .
$$

Note that $g_{c}^{2} \ll 1$ for the very massive states $\left(M \gg M_{s}\right)$ that we consider. This justifies our use of the perturbative relation between $G_{N}$ and $\alpha^{\prime}$.

In the case of extremal BPS, and nearly extremal, black holes the conjectured correspondence was dramatically confirmed through the work of Strominger and Vafa [10] and others 11] leading to a statistical mechanics interpretation of black-hole entropy in terms of the number of microscopic states sharing the same macroscopic quantum numbers. However, little is known about whether and how the correspondence works for non-extremal, non BPS black holes, such as the simplest Schwarzschild black hole By contrast to BPS states whose mass is protected by supersymmetry, we shall consider here the effect of varying $g$ on the mass and size of non-BPS string states.

\footnotetext{
${ }^{\mathrm{c}}$ One uses here the fact that, during an adiabatic variation of $g$, the entropy of the black hole $S_{\mathrm{BH}} \sim\left(\right.$ Area) $/ G_{N} \sim R_{\mathrm{BH}}^{d-1} / G_{N}$ stays constant. This result (known to hold in the Einstein conformal frame) applies also in string units because $S_{\mathrm{BH}}$ is dimensionless.

d'The variation of $g$ can be seen, depending on one's taste, either as a real, adiabatic change of $g$ due to a varying dilaton background, or as a mathematical way of following energy states.

eFor simplicity, we shall consider in this work only Schwarzschild black holes, in any number $d \equiv D-1$ of non-compact spatial dimensions.
} 
Although it is remarkable that black-hole and string entropy coincide when $R_{\mathrm{BH}}=$ $\ell_{s}$, this is still not quite sufficient to claim that, when starting from a string state, a string becomes a black hole at $g=g_{c}$. In fact, the process in which one starts from a string state in flat space and increases $g$ poses a serious puzzle [6]. Indeed, the radius of a typical excited string state of mass $M$ is generally thought of being of order

$$
R_{s}^{\mathrm{rw}} \sim \ell_{s}\left(M / M_{s}\right)^{1 / 2},
$$

as if a highly excited string state were a random walk made of $M / M_{s}=\alpha^{\prime} M / \ell_{s}$ segments of length $\ell_{s}$ 12. [The number of steps in this random walk is, as is natural, the string entropy (11).] The "random walk" radius (6) is much larger than the Schwarzschild radius for all couplings $g \leq g_{c}$, or, equivalently, the ratio of selfgravitational binding energy to mass (in $d$ spatial dimensions)

$$
\frac{G_{N} M}{\left(R_{s}^{\mathrm{rw}}\right)^{d-2}} \sim\left(\frac{R_{\mathrm{BH}}(M)}{R_{s}^{\mathrm{rw}}}\right)^{d-2} \sim g^{2}\left(\frac{M}{M_{s}}\right)^{\frac{4-d}{2}}
$$

remains much smaller than one (when $d>2$, to which we restrict ourselves) up to, and including, the transition point. In view of (7) it does not seem natural to expect that a string state will "collapse" to a black hole when $g$ reaches the value (四). One would expect a string state of mass $M$ to turn into a black hole only when its typical size is of order of $R_{\mathrm{BH}}(M)$ (which is of order $\ell_{s}$ at the expected transition point (何). According to Eq. (7), this seems to happen for a value of $g$ much larger than $g_{c}$.

Horowitz and Polchinski [13] have addressed this puzzle by means of a "thermal scalar" formalism [14]. Their results suggest a resolution of the puzzle when $d=3$ (four-dimensional spacetime), but lead to a rather complicated behaviour when $d \geq 4$. Moreover, even in the simple $d=3$ case, the formal nature of the auxiliary "thermal scalar" renders unclear (at least to me) the physical interpretation of their analysis.

Here, I will review the results of a recent collaboration with G. Veneziano 15 whose aim was to clarify the string $\rightleftharpoons$ black hole transition by a direct study, in real spacetime, of the size and mass of a typical excited string, within the microcanonical ensemble of self-gravitating strings. Our results 15 lead to a rather simple picture of the transition, in any dimension. We find no hysteresis phenomenon in higher dimensions. The critical value for the transition is (位), or (5) in terms of the entropy $S$, for both directions of the string $\rightleftharpoons$ black hole transition. In three spatial dimensions, we find that the size (computed in real spacetime) of a typical self-gravitating string is given by the random walk value (6) when $g^{2} \leq g_{0}^{2}$, with $g_{0}^{2} \sim\left(M / M_{s}\right)^{-3 / 2} \sim S^{-3 / 2}$, and by

$$
R_{\text {typ }} \sim \frac{1}{g^{2} M},
$$

when $g_{0}^{2} \leq g^{2} \leq g_{c}^{2}$. Note that $R_{\text {typ }}$ smoothly interpolates between $R_{s}^{\mathrm{rw}}$ and $\ell_{s}$. This result confirms the picture proposed by Ref. [13] when $d=3$, but with the bonus that Eq. (8) refers to a radius which is estimated directly in physical space (see below), and which is the size of a typical member of the microcanonical ensemble of self-gravitating strings. In all higher dimensions 5 , we find that the size of a typical self-gravitating

\footnotetext{
${ }^{\mathrm{f}}$ With the proviso that the consistency of our analysis is open to doubt when $d \geq 8$.
} 
string remains fixed at the random walk value (6) when $g \leq g_{c}$. However, when $g$ gets close to a value of order $g_{c}$, the ensemble of self-gravitating strings becomes (smoothly in $d=4$, but suddenly in $d \geq 5$ ) dominated by very compact strings of size $\sim \ell_{s}$ (which are then expected to collapse with a slight further increase of $g$ because the dominant size is only slightly larger than the Schwarzschild radius at $g_{c}$ ).

Our results 15 confirm and clarify the main idea of a correspondence between string states and black hole states [6], [8], [9], [13], and suggest that the transition between these states is rather smooth, with no apparent hysteresis, and with continuity in entropy, mass, typical size, and luminosity. It is, however, beyond the technical grasp of our analysis to compute any precise number at the transition (such as the famous factor $1 / 4$ in the Bekenstein-Hawking entropy formula).

\section{Size distribution of free string states}

For simplicity, we deal with open bosonic strings $\left(\ell_{s} \equiv \sqrt{2 \alpha^{\prime}}, 0 \leq \sigma \leq \pi\right)$

$$
\begin{aligned}
& X^{\mu}(\tau, \sigma)=X_{\mathrm{cm}}^{\mu}(\tau, \sigma)+\widetilde{X}^{\mu}(\tau, \sigma), \\
& X_{\mathrm{cm}}^{\mu}(\tau, \sigma)=x^{\mu}+2 \alpha^{\prime} p^{\mu} \tau, \\
& \widetilde{X}^{\mu}(\tau, \sigma)=i \ell_{s} \sum_{n \neq 0} \frac{\alpha_{n}^{\mu}}{n} e^{-i n \tau} \cos n \sigma .
\end{aligned}
$$

Here, we have explicitly separated the center of mass motion $X_{\mathrm{cm}}^{\mu}$ (with $\left[x^{\mu}, p^{\nu}\right]=$ $\left.i \eta^{\mu \nu}\right)$ from the oscillatory one $\widetilde{X}^{\mu}\left(\left[\alpha_{m}^{\mu}, \alpha_{n}^{\nu}\right]=m \delta_{m+n}^{0} \eta^{\mu \nu}\right)$. The free spectrum is given by $\alpha^{\prime} M^{2}=N-1$ where $\left(\alpha \cdot \beta \equiv \eta_{\mu \nu} \alpha^{\mu} \beta^{\nu} \equiv-\alpha^{0} \beta^{0}+\alpha^{i} \beta^{i}\right)$

$$
N=\sum_{n=1}^{\infty} \alpha_{-n} \cdot \alpha_{n}=\sum_{n=1}^{\infty} n N_{n}
$$

Here $N_{n} \equiv a_{n}^{\dagger} \cdot a_{n}$ is the occupation number of the $n^{\text {th }}$ oscillator $\left(\alpha_{n}^{\mu}=\sqrt{n} a_{n}^{\mu},\left[a_{n}^{\mu}, a_{m}^{\nu \dagger}\right]=\right.$ $\eta^{\mu \nu} \delta_{n m}$, with $n, m$ positive).

The decomposition (9)-(11) holds in any conformal gauge $\left(\left(\partial_{\tau} X^{\mu} \pm \partial_{\sigma} X^{\mu}\right)^{2}=0\right)$. One can further specify the choice of worldsheet coordinates by imposing

$$
n_{\mu} X^{\mu}(\tau, \sigma)=2 \alpha^{\prime}\left(n_{\mu} p^{\mu}\right) \tau,
$$

where $n^{\mu}$ is an arbitrary timelike or null vector $(n \cdot n \leq 0)$ [16. Eq. (13) means that the $n$-projected oscillators $n_{\mu} \alpha_{m}^{\mu}$ are set equal to zero. As we shall be interested in quasi-classical, very massive string states $(N \gg 1)$ it should be possible to work in the "center of mass" gauge, where the vector $n^{\mu}$ used in Eq. (13) to define the $\tau$-slices of the world-sheet is taken to be the total momentum $p^{\mu}$ of the string. This gauge is the most intrinsic way to describe a string in the classical limit. Using this intrinsic gauge, one can covariantly define the proper rms size of a massive string state as

$$
R^{2} \equiv \frac{1}{d}\left\langle\left(\widetilde{X}_{\perp}^{\mu}(\tau, \sigma)\right)^{2}\right\rangle_{\sigma, \tau},
$$


where $\widetilde{X}_{\perp}^{\mu} \equiv \widetilde{X}^{\mu}-p^{\mu}(p \cdot \widetilde{X}) /(p \cdot p)$ denotes the projection of $\tilde{X}^{\mu} \equiv X^{\mu}-X_{\mathrm{cm}}^{\mu}(\tau)$ orthogonally to $p^{\mu}$, and where the angular brackets denote the (simple) average with respect to $\sigma$ and $\tau$.

In the center of mass gauge, $p_{\mu} \widetilde{X}^{\mu}$ vanishes by definition, and Eq. (14) yields simply

$$
R^{2}=\frac{1}{d} \ell_{s}^{2} \mathcal{R}
$$

with (after discarding a logarithmically infinite, but state independent, contribution)

$$
\mathcal{R} \equiv \sum_{n=1}^{\infty} \frac{\alpha_{-n} \cdot \alpha_{n}}{n^{2}}=\sum_{n=1}^{\infty} \frac{a_{n}^{\dagger} \cdot a_{n}}{n}=\sum_{n=1}^{\infty} \frac{N_{n}}{n} .
$$

We wish to estimate the distribution function in size of the ensemble of free string states of mass $M$, i.e. to count the number of string states, having some fixed values of $M$ and $R$ (or, equivalently, $N$ and $\mathcal{R}$ ). An approximate estimate of this number ("degeneracy") is 15

$$
\mathcal{D}(M, R) \sim \exp \left[c(R) a_{0} M\right],
$$

where $a_{0}=2 \pi\left((d-1) \alpha^{\prime} / 6\right)^{1 / 2}$ and

$$
c(R)=\left(1-\frac{c_{1}}{R^{2}}\right)\left(1-c_{2} \frac{R^{2}}{M^{2}}\right)
$$

with the coefficients $c_{1}$ and $c_{2}$ being of order unity in string units. The coefficient $c(R)$ gives the fractional reduction in entropy brought by imposing a size constraint. Note that (as expected) this reduction is minimized when $c_{1} R^{-2} \sim c_{2} R^{2} / M^{2}$, i.e. for $R \sim R_{\mathrm{rw}} \sim \ell_{s} \sqrt{M / M_{s}}$.

\section{Mass shift of string states due to self-gravity}

We also need to estimate the mass shift of string states (of mass $M$ and size $R$ ) due to the exchange of the various long-range fields which are universally coupled to the string: graviton, dilaton and axion. As we are interested in very massive string states, $M \gg M_{s}$, in extended configurations, $R \gg \ell_{s}$, we expect that massless exchange dominates the (state-dependent contribution to the) mass shift. [The exchange of spin 1 fields (for open strings) becomes negligible when $M \gg M_{s}$ because it does not increase with $M$.]

The evaluation, in string theory, of (one loop) mass shifts for massive states is technically quite involved, and can only be tackled for the states which are near the leading Regge trajectory [19]. [Indeed, the vertex operators creating these states are the only ones to admit a manageable explicit oscillator representation.] As we consider states which are very far from the leading Regge trajectory, there is no hope of computing exactly (at one loop) their mass shifts.

In Ref. [15] we could estimate the one-loop mass-shift by resorting to a semiclassical approximation. The starting point of this semi-classical approximation is 
the effective action of self-gravitating fundamental strings derived in Ref. 20]. Using coherent-state methods [21], [16], 22] and a generalization of Bloch's theorem (see Eq. (3.13) of [15]) one finds

$$
\delta M \simeq-c_{d} G_{N} \frac{M^{2}}{R^{d-2}},
$$

with the (positive) numerical constant

$$
c_{d}=\left[\frac{d-2}{2}(4 \pi)^{\frac{d-2}{2}}\right]^{-1},
$$

equal to $1 / \sqrt{\pi}$ in $d=3$.

The result (19) was expected in order of magnitude, but it is important to check that it approximately comes out of a detailed calculation of the mass shift which incorporates both relativistic and quantum effects and which uses the precise definition (16) of the squared size.

Finally, let us mention that, by using the same tools Ref. 15 has computed the imaginary part of the mass shift $\delta M=\delta M_{\text {real }}-i \Gamma / 2$, i.e. the total decay rate $\Gamma$ in massless quanta, as well as the total power radiated $P$. In order of magnitude these quantities are

$$
\Gamma \sim g^{2} M, P \sim g^{2} M M_{s}
$$

\section{Entropy of self-gravitating strings}

Finally one combines the results of the previous sections, Eqs. (17) and (19), and heuristically extend them at the limit of their domain of validity. We consider a narrow band of string states that we follow when increasing adiabatically the string coupling $g$, starting from $g=0$. Let $M_{0}, R_{0}$ denote the "bare" values (i.e. for $g \rightarrow 0$ ) of the mass and size of this band of states. Under the adiabatic variation of $g$, the mass and size, $M, R$, of this band of states will vary. However, the entropy $S(M, R)$ remains constant under this adiabatic process: $S(M, R)=S\left(M_{0}, R_{0}\right)$. We consider states with sizes $\ell_{s} \ll R_{0} \ll M_{0}$ for which the correction factor,

$$
c\left(R_{0}\right) \simeq\left(1-c_{1} R_{0}^{-2}\right)\left(1-c_{2} R_{0}^{2} / M_{0}^{2}\right)
$$

in the entropy

$$
S\left(M_{0}, R_{0}\right)=c\left(R_{0}\right) a_{0} M_{0}
$$

is near unity. [We use Eq. (17) in the limit $g \rightarrow 0$, for which it was derived.] Because of this reduced sensitivity of $c\left(R_{0}\right)$ on a possible direct effect of $g$ on $R$ (i.e. $R(g)=$ $R_{0}+\delta_{g} R$ ), the main effect of self-gravity on the entropy (considered as a function of the actual values $M, R$ when $g \neq 0$ ) will come from replacing $M_{0}$ as a function of $M$ 
and $R$. The mass-shift result (19) gives $\delta M=M-M_{0}$ to first order in $g^{2}$. To the same accuracy , (19) gives $M_{0}$ as a function of $M$ and $R$ :

$$
M_{0} \simeq M+c_{3} g^{2} \frac{M^{2}}{R^{d-2}}=M\left(1+c_{3} \frac{g^{2} M}{R^{d-2}}\right),
$$

where $c_{3}$ is a positive numerical constant.

Finally, combining Eqs. (22)-(24) (and neglecting, as just said, a small effect linked to $\delta_{g} R \neq 0$ ) leads to the following relation between the entropy, the mass and the size (all considered for self-gravitating states, with $g \neq 0$ )

$$
S(M, R) \simeq a_{0} M\left(1-\frac{1}{R^{2}}\right)\left(1-\frac{R^{2}}{M^{2}}\right)\left(1+\frac{g^{2} M}{R^{d-2}}\right) .
$$

For notational simplicity, we henceforth set to unity (by using suitable redefinitions) the coefficients $c_{1}, c_{2}$ and $c_{3}$. The possibility of smoothly transforming self-gravitating string states into black hole states come from the peculiar radius dependence of the entropy $S(M, R)$. Eq. (24) exhibits two effects varying in opposite directions: (i) selfgravity favors small values of $R$ (because they correspond to larger values of $M_{0}$, i.e. of the "bare" entropy), and (ii) the constraint of being of some fixed size $R$ disfavors both small $(R \ll \sqrt{M})$ and large $(R \gg \sqrt{M})$ values of $R$. For given values of $M$ and $g$, the most numerous (and therefore most probable) string states will have a size $R_{*}(M ; g)$ which maximizes the entropy $S(M, R)$. Said differently, the total degeneracy of the complete ensemble of self-gravitating string states with total energy $M$ (and no a priori size restriction) will be given by an integral (where $\Delta R$ is the rms fluctuation of $R$ )

$$
\mathcal{D}(M) \sim \int \frac{d R}{\Delta R} e^{S(M, R)} \sim e^{S\left(M, R_{*}\right)}
$$

which will be dominated by the saddle point $R_{*}$ which maximizes the exponent.

The value of the most probable size $R_{*}$ is a function of $M, g$ and the space dimension $d$. We refer to Ref. [15] for a full treatment. Let us only indicate the results in the (actual) case where $d=3$. When maximizing the entropy $S(M, R)$ with respect to $R$ one finds that: (i) when $g^{2} \ll M^{-3 / 2}$, the most probable size $R_{*}(M, g) \sim \sqrt{M}$, (ii) when $g^{2} \gg M^{-3 / 2}$,

$$
R_{*}(M, g) \simeq \frac{1+\sqrt{1+3 \lambda^{2}}}{\lambda}
$$

where $\lambda \equiv g^{2} M$.

Eq. (27) says that, when $g^{2}$ increases, and therefore when $\lambda$ increases (beyond $M^{-1 / 2}$ ) the typical size of a self-gravitating string decreases, and (formally) tends to a limiting size of order unity, $R_{\infty}=\sqrt{3}$ (i.e. of order the string length scale $\ell_{s}=\sqrt{2 \alpha^{\prime}}$ ) when $\lambda \gg 1$. However, the fractional self-gravity $G_{N} M / R_{*} \simeq \lambda / R_{*}$ (which measures the gravitational deformation away from flat space) becomes unity for $\lambda=\sqrt{5}$ and formally increases without limit when $\lambda$ further increases. Therefore, we expect that

\footnotetext{
g Actually, Eq. 24) is probably a more accurate version of the mass-shift formula because it exhibits the real mass $M$ (rather than the bare mass $M_{0}$ ) as the source of self-gravity.
} 
for some value of $\lambda$ of order unity, the self-gravity of the compact string state already reached when $\lambda \sim 1$ (indeed, Eq. (27) predicts $R_{*} \sim 1$ when $\lambda \sim 1$ ) will become so strong that it will (continuously) turn into a black hole state. Having argued that the dynamical threshold for the transition string $\rightarrow$ black hole is $\lambda \sim 1$, we now notice that, for such a value of $\lambda$ the entropy $S(M)=S\left(M, R_{*}(M)\right) \simeq a_{0} M\left[1+\frac{1}{4}\left(g^{2} M\right)^{2}\right]$ of the string state (of mass $M$ ) matches the Bekenstein-Hawking entropy $S_{\mathrm{BH}}(M) \sim g^{2} M^{2}=$ $\lambda M$ of the formed black hole. One further checks that the other global physical characteristics of the string state (radius $R_{*}$, luminosity $P$, Eq. (21)) match those of a Schwarzschild black hole of the same mass $\left(R_{\mathrm{BH}} \sim G M \sim g^{2} M, P_{\text {Hawking }} \sim R_{\mathrm{BH}}^{-2}\right)$ when $\lambda \sim 1$.

\section{Discussion}

Conceptually, the main new result of this paper concerns the most probable state

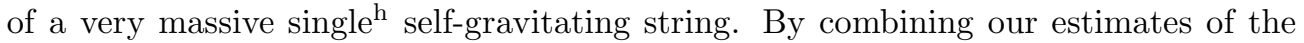
entropy reduction due to the size constraint, and of the mass shift we come up with the expression (25) for the logarithm of the number of self-gravitating string states of size $R$. Our analysis of the function $S(M, R)$ clarifies the correspondence [6], [8], [9], 13] between string states and black holes. In particular, our results confirm many of the results of [13], but make them (in our opinion) physically clearer by dealing directly with the size distribution, in real space, of an ensemble of string states. When our results differ from those of [13], they do so in a way which simplifies the physical picture and make even more compelling the existence of a correspondence between strings and black holes. The simple physical picture suggested by our results is the following: In any dimension, if we start with a massive string state and increase the string coupling $g$, a typical string state will, eventually, become more compact and will end up, when $\lambda_{c}=g_{c}^{2} M \sim 1$, in a "condensed state" of size $R \sim 1$, and mass density $\rho \sim g_{c}^{-2}$. Note that the basic reason why small strings, $R \sim 1$, dominate in any dimension the entropy when $\lambda \sim 1$ is that they descend from string states with bare mass $M_{0} \simeq M\left(1+\lambda / R^{d-2}\right) \sim 2 M$ which are exponentially more numerous than less condensed string states corresponding to smaller bare masses.

The nature of the transition between the initial "dilute" state and the final "condensed" one depends on the value of the space dimension $d$. In $d=3$, the transition is gradual: when $\lambda<M^{-1 / 2}$ the size of a typical state is $R_{*}^{(d=3)} \simeq M^{1 / 2}\left(1-M^{1 / 2} \lambda / 8\right)$, when $\lambda>M^{1 / 2}$ the typical size is $R_{*}^{(d=3)} \simeq\left(1+\left(1+3 \lambda^{2}\right)^{1 / 2}\right) / \lambda$. In $d=4$, the transition toward a condensed state is still continuous, but most of the size evolution takes place very near $\lambda=1$ : when $\lambda<1, R_{*}^{(d=4)} \simeq M^{1 / 2}(1-\lambda)^{1 / 4}$, and when $\lambda>1, R_{*}^{(d=4)} \simeq(2 \lambda /(\lambda-1))^{1 / 2}$, with some smooth blending between the two evolu-

\footnotetext{
${ }^{\mathrm{h}}$ We consider states of a single string because, for large values of the mass, the single-string entropy approximates the total entropy up to subleading terms.

${ }^{\mathrm{i}}$ Our conclusions are not rigourously established because they rely on assuming the validity of the result (25) beyond the domain $\left(R^{-2} \ll 1, g^{2} M / R^{d-2} \ll 1\right)$ where it was derived. However, we find heuristically convincing to believe in the presence of a reduction factor of the type $1-R^{-2}$ down to sizes very near the string scale. Our heuristic dealing with self-gravity is less compelling because we do not have a clear signal of when strong gravitational field effects become essential.
} 
tions around $|\lambda-1| \sim M^{-2 / 3}$. In $d \geq 5$, the transition is discontinuous (like a first order phase transition between, say, gas and liquid states). Barring the consideration of metastable (supercooled) states, on expects that when $\lambda=\lambda_{2} \simeq \nu^{\nu} /(\nu-1)^{\nu-1}$ (with $\nu=(d-2) / 2$ ), the most probable size of a string state will jump from $R_{\mathrm{rw}}$ (when $\lambda<\lambda_{2}$ ) to a size of order unity (when $\lambda>\lambda_{2}$ ).

One can think of the "condensed" state of (single) string matter, reached (in any d) when $\lambda \sim 1$, as an analog of a neutron star with respect to an ordinary star (or a white dwarf). It is very compact (because of self gravity) but it is stable (in some range for $g$ ) under gravitational collapse. However, if one further increases $g$ or $M$ (in fact, $\lambda=g^{2} M$ ), the condensed string state is expected (when $\lambda$ reaches some $\lambda_{3}>\lambda_{2}, \lambda_{3}=\mathcal{O}(1)$ ) to collapse down to a black hole state (analogously to a neutron star collapsing to a black hole when its mass exceeds the Landau-Oppenheimer-Volkoff critical mass). Still in analogy with neutron stars, one notes that general relativistic strong gravitational field effects are crucial for determining the onset of gravitational collapse; indeed, under the "Newtonian" approximation (25), the condensed string state could continue to exist for arbitrary large values of $\lambda$.

It is interesting to note that the value of the mass density at the formation of the condensed string state is $\rho \sim g^{-2}$. This is reminiscent of the prediction by Atick and Witten [23] of a first-order phase transition of a self-gravitating thermal gas of strings, near the Hagedorn temperature], towards a dense state with energy density $\rho \sim g^{-2}$ (typical of a genus-zero contribution to the free energy). Ref. 23 suggested that this transition is first-order because of the coupling to the dilaton. This suggestion agrees with our finding of a discontinuous transition to the single string condensed state in dimensions $\geq 5$ (Ref. 23 work in higher dimensions, $d=25$ for the bosonic case). It would be interesting to deepen these links between self-gravitating single string states and multi-string states.

Let us come back to the consequences of the picture brought by the present work for the problem of the end point of the evaporation of a Schwarzschild black hole and the interpretation of black hole entropy. In that case one fixes the value of $g$ (assumed to be $\ll 1$ ) and considers a black hole which slowly looses its mass via Hawking radiation. When the mass gets as low as a value $M \sim g^{-2}$, for which the radius of the black hole is of order one (in string units), one expects the black hole to transform (in all dimensions) into a typical stringl] state corresponding to $\lambda=g^{2} M \sim 1$, which is a dense state (still of radius $R \sim 1$ ). This string state will further decay and loose mass, predominantly via the emission of massless quanta, with a quasi thermal spectrum with temperature $T \sim T_{\text {Hagedorn }}=a_{0}^{-1}$ which smoothly matches the previous black hole Hawking temperature. This mass loss will further decrease the product $\lambda=g^{2} M$, and this decrease will, either gradually or suddenly, cause the initially compact string state to inflate to much larger sizes. For instance, if $d \geq 4$, the string state will

\footnotetext{
${ }^{\mathrm{j} N o t e}$ that, by definition, in our single string system, the formal temperature $T=(\partial S / \partial M)^{-1}$ is always near the Hagedorn temperature.

${ }^{\mathrm{k}}$ Note that the mass at the black hole $\rightarrow$ string transition is larger than the Planck mass $M_{P} \sim$ $\left(G_{N}\right)^{-1 / 2} \sim g^{-1}$ by a factor $g^{-1} \gg 1$.

${ }^{1}$ A check on the single-string dominance of the transition black hole $\rightarrow$ string is to note that the single string entropy $\sim M / M_{s}$ is much larger than the entropy of a ball of radiation $S_{\text {rad }} \sim$ $(R M)^{d /(d+1)}$ with size $R \sim R_{\mathrm{BH}} \sim \ell_{s}$ at the transition.
} 
quickly inflate to a size $R \sim \sqrt{M}$. Later, with continued mass loss, the string size will slowly shrink again toward $R \sim 1$ until a remaining string of mass $M \sim 1$ finally decays into stable massless quanta. In this picture, the black hole entropy acquires a somewhat clear statistical significance (as the degeneracy of a corresponding typical string state) only when $M$ and $g$ are related by $g^{2} M \sim 1$. If we allow ourselves to vary (in a Gedanken experiment) the value of $g$ this gives a potential statistical significance to any black hole entropy value $S_{\mathrm{BH}}$ (by choosing $g^{2} \sim S_{\mathrm{BH}}^{-1}$ ). We do not claim, however, to have a clear idea of the direct statistical meaning of $S_{\mathrm{BH}}$ when $g^{2} S_{\mathrm{BH}} \gg 1$. Neither do we clearly understand the fate of the very large space (which could be excited in many ways) which resides inside very large classical black holes of radius $R_{\mathrm{BH}} \sim\left(g^{2} S_{\mathrm{BH}}\right)^{1 /(d-1)} \gg 1$. The fact that the interior of a black hole of given mass could be arbitrarily large $\mathrm{m}$, and therefore arbitrarily complex, suggests that black hole physics is not exhausted by the idea (confirmed in the present paper) of a reversible transition between string-length-size black holes and string states.

On the string side, we also do not clearly understand how one could follow in detail (in the present non BPS framework) the "transformation" of a strongly self-gravitating string state into a black hole state.

Finally, let us note that we expect that self-gravity will lift nearly completely the degeneracy of string states. [The degeneracy linked to the rotational symmetry, i.e. $2 J+1$ in $d=3$, is probably the only one to remain, and it is negligible compared to the string entropy.] Therefore we expect that the separation $\delta E$ between subsequent (string and black hole) energy levels will be exponentially small: $\delta E \sim \Delta M \exp (-S(M))$, where $\Delta M$ is the canonical-ensemble fluctuation in $M$. Such a $\delta E$ is negligibly small compared to the radiative width $\Gamma \sim g^{2} M$ of the levels. This seems to mean that the discreteness of the quantum levels of strongly self-gravitating strings and black holes is very much blurred, and difficult to see observationally.

\section{References}

[1] S. Fubini and G. Veneziano, Nuovo Cim. 64 A 811 (1969);

K.Huang and S. Weinberg, Phys. Rev. Lett 25895 (1970).

[2] J. D. Bekenstein, Phys. Rev. D 72333 (1973).

[3] S. W. Hawking, Comm. Math. Phys. 43199 (1975).

[4] M. Bowick, L. Smolin and L.C.R. Wijewardhana, Gen. Rel. Grav. 19113 (1987).

[5] G. Veneziano, Europhys. Lett. 2199 (1986).

6 L. Susskind, hep-th/9309145 (unpublished).

[7] G. Veneziano, in Hot Hadronic Matter: Theory and Experiments, Divonne, June 1994, eds. J. Letessier, H. Gutbrod and J. Rafelsky, NATO-ASI Series B: Physics, 346 (Plenum Press, New York 1995), p. 63.

[8] E. Halyo, A. Rajaraman and L. Susskind, Phys. Lett. B 392, 319 (1997);

E.Halyo, B. Kol, A. Rajaraman and L. Susskind, Phys. Lett. B 401,15 (1997).

[9] G.T. Horowitz and J. Polchinski, Phys. Rev. D 55, 6189 (1997).

[10] A. Strominger and C. Vafa, Phys. Lett. B 37999 (1996).

[11] E.g. A. Sen, Mod. Phys. Lett. A 102081 (1995);

C.G. Callan and J.M. Maldacena, Nucl. Phys. B 472, 591 (1996);

J.C. Breckenridge et al. Phys. Lett. B 381423 (1996).

\footnotetext{
${ }^{\mathrm{m}}$ E.g., in the Oppenheimer-Snyder model, one can join an arbitrarily large closed Friedmann dust universe, with hyperspherical opening angle $0 \leq \chi_{0} \leq \pi$ arbitrarily near $\pi$, onto an exterior Schwarzschild spacetime of given mass $M$.
} 
[12] P. Salomonson and B.S. Skagerstam, Nucl. Phys. B 268,349 (1986);

Physica A 158, 499 (1989);

D. Mitchell and N. Turok, Phys. Rev. Lett. 58, 1577 (1987);

Nucl.Phys. B 294, 1138 (1987).

[13] G.T. Horowitz and J. Polchinski, Phys. Rev. D 57,2557 (1998).

[14] B. Sathiapalan, Phys. Rev. D 35, 3277 (1987);

I.A. Kogan, JETP Lett. 45, 709 (1987);

J.J. Atick and E. Witten, Nucl. Phys. B 310, 291 (1988).

[15] T. Damour and G. Veneziano, Nucl. Phys. B, in press; hep-th/9907030.

[16] J. Scherk, Rev. Mod. Phys. 47, 123 (1975).

[17] M. Karliner, I. Klebanov and L. Susskind, Int. J. Mod.Phys. A3, 1981 (1988).

[18] L.D. Landau and E.M. Lifshitz, Statistical Physics, part 1, third edition (Pergamon Press, Oxford, 1980).

[19] H. Yamamoto, Prog. Theor. Phys. 79, 189 (1988);

K. Amano and A. Tsuchiya, Phys. Rev. D 39, 565 (1989);

B. Sundborg, Nucl. Phys. B 319, 415 (1989); and 338, 101 (1990).

[20] A. Buonanno and T. Damour, Phys. Lett. B 432, 51(1998).

[21] V. Alessandrini, D. Amati, M. Le Bellac and D. Olive, Phys. Rep. C1, 269 (1971).

[22] M.B. Green, J.H. Schwarz and E. Witten, Superstring Theory, Volume 1, (Cambridge University Press, Cambridge, 1987).

[23] J.J. Atick and E. Witten, Nucl. Phys. B 310, 291(1988). 